\title{
La cultura popular para la enseñanza de español como lengua extranjera*
}

\author{
WILSON EDUARDO ROJAS MORENO**
}

Recepción: 08 de agosto de 2018

Aprobación: 13 de junio de 2019

Forma de citar este artículo: Rojas, W. E. (2019). La cultura popular para la enseñanza de español como lengua extranjera. Cuadernos de Lingüística Hispánica, (34), pp 177-193.

(doi) $10.19053 / 0121053 X . n 34.2019 .9373$

* Artículo de investigación, enmarcado dentro del proyecto de investigación "Desarrollo de la competencia comunicativa a través de la cultura popular para el aprendizaje del español como lengua extranjera (ele), del grupo de investigación ELEX, de la Universidad Pedagógica y Tecnológica de Colombia.

** Estudiante de decimo semestre de la Licenciatura en Lenguas Extranjeras de la Universidad Pedagógica y Tecnológica de Colombia. Miembro del grupo de investigación ELEX, de la Universidad Pedagógica y Tecnológica de Colombia. Correo electrónico: wilson.rojas02@uptc.edu.co. (B) https://orcid.org/0000-0001-9690-1177 


\section{Resumen}

Este artículo presenta diversos aspectos de una investigación sobre la enseñanza de español como lengua extranjera (ELE). El proyecto se enfocó en el uso de la cultura popular colombiana para enseñar español a un grupo de extranjeros en la Universidad Pedagógica y Tecnológica de Colombia, utilizando diferentes textos narrativos y populares: el mito, la leyenda, el refrán y el agüero. El propósito de este proyecto fue diseñar y aplicar una cartilla, que comprendió diversos textos narrativos con ejercicios y actividades. En relación con la metodología, el enfoque de la investigación fue cualitativo, en la medida en que tanto para la recolección y el análisis de información como para el diseño de las unidades, se tuvo en cuenta la información que arrojó el pilotaje, que permitió la concepción de la propuesta didáctica. Como resultado se produjo la cartilla intitulada Enseñanza del español para extranjeros a través de la cultura popular, que recopila doce unidades que fueron desarrolladas en su totalidad por los estudiantes extranjeros. Se concluyó que el uso de textos narrativos (el mito, la leyenda, el refrán y el agüero) permitió a los aprendientes perfeccionar su nivel de comprensión y expresión ampliando al mismo tiempo su conocimiento de la cultura colombiana.

Palabras clave: leyenda, mito, cultura popular, enseñanza, ELE (español como lengua extranjera).

\section{Popular Culture to Teach Spanish as a Foreign Language}

\section{Abstract}

This paper presents various aspects of a research on teaching Spanish as a foreign language (ELE, by its acronym in Spanish). The project focused on the use of Colombian popular culture to teach Spanish to a group of foreigners at Universidad Pedagógica y Tecnológica de Colombia, using different narrative and popular texts: the myth, the legend, the proverb and the omen. The purpose of this project was to design and apply a primer, which included some narrative texts with exercises and activities. In relation to the methodology, the research's focus was qualitative, insofar as both the collection and analysis of information and the units' design took into account the information provided by the piloting, which allowed the conception of the didactic proposal. As a result, the book Teaching Spanish for foreigners through popular culture was produced, which compiles twelve units that were developed entirely by foreign students. It was concluded that the use of narrative texts (the myth, the legend, the proverb and the omen) allowed the learners to improve their levels of comprehension and expression while expanding their knowledge of Colombian culture.

Keywords: legend, myth, popular culture, teaching, ELE (Spanish as a foreign language). 


\section{La culture populaire pour l'enseignement de l'espagnol comme langue étrangère}

\section{Résumé}

Cet article présente de divers aspects d'une recherche sur l'enseignement de l'espagnol comme langue étrangère (ELE). Le projet a été focalisé sur l'utilisation de la culture populaire colombienne pour enseigner l'espagnol à un groupe d'étrangers à l'Université Pedagógica y Tecnológica de Colombia, en utilisant de différents textes narratifs et populaire : le mythe, la légende, le proverbe et le présage. Le propos de ce projet a été celui de concevoir et mettre en pratique un livret, contenant de divers textes narratifs avec des exercices et des activités. Par rapport à la méthodologie, l'orientation de la recherche a été qualitative, car tant pour la collecte et l'analyse de l'information, que pour le design des unités, on a pris en considération l'information fournie par le pilotage, ce qui a permis la conception de la proposition didactique. Comme résultat, on a un livret intitulé Enseignement de l'espagnol pour étrangers à travers la culture populaire, qui recueille douze unités, développées complètement par les étudiants étrangers. On a conclu que l'utilisation de textes narratifs (le mythe, la légende, le proverbe et le présage) a permis aux apprenants de perfectionner leur niveau de compréhension et expression, en élargissant en même temps, leur connaissance de la culture colombienne.

Mots clés: légende, mythe, culture populaire, enseignement, ELE (espagnol comme langue étrangère).

\section{Cultura popular do ensino em espanhol como língua estrangeira}

\section{Resumo}

Este artigo apresenta vários aspectos de uma investigação sobre o ensino do espanhol como língua estrangeira (ELE). 0 projeto focou no uso da cultura popular colombiana para ensinar espanhol a um grupo de estrangeiros da Universidade Pedagógica e Tecnológica da Colômbia, usando diferentes textos narrativos e populares: mito, lenda, provérbio e presságio. 0 objetivo deste projeto foi projetar e aplicar um livreto, que incluía vários textos narrativos com exercícios e atividades. Em relação à metodologia, a abordagem de pesquisa foi qualitativa, tanto na coleta e análise de informações quanto no desenho das unidades, foram consideradas as informações produzidas pelo piloto, o que permitiu a concepção da proposta didática. Como resultado, o livreto intitulado Ensinar espanhol para estrangeiros foi produzido através da cultura popular, que reúne doze unidades que foram desenvolvidas inteiramente por estudantes estrangeiros. Concluiu-se que o uso de textos narrativos (o mito, a lenda, o ditado e 0 presságio) permitiu que os alunos melhorassem seu nível de compreensão e expressão enquanto expandiam seus conhecimentos sobre a cultura colombiana.

Palavras-chave: lenda, mito, cultura popular, ensino, ELE (espanhol como língua estrangeira). 


\section{Introducción}

El español es la lengua materna de más de 500 millones de personas y la segunda lengua de comunicación mundial. En los últimos tiempos ha venido aumentando la cantidad de personas interesadas en aprender español en los distintos países donde se utiliza como primera lengua, debido a la influencia global en cuanto a cultura, turismo, economía o educación. Por eso se promueve la oferta de cursos de español como lengua extranjera (ELE) no solamente en el ámbito internacional, sino también regional y local.

En cuanto al contexto boyacense, cada día llegan más visitantes y estudiantes extranjeros a la ciudad de Tunja y a la Universidad Pedagógica y Tecnológica de Colombia, gracias a convenios, pasantías, intercambios o negocios. De ahí la necesidad de la contextualización de los aspectos culturales con el fin de promover el desarrollo de investigaciones que tengan como punto central las metodologías y contenidos apropiados para la enseñanza y el aprendizaje del español como lengua extranjera. Teniendo en cuenta lo anterior, el grupo de investigación Español como Lengua Extrajera, cuyas siglas son ELEX, adscrito a la Universidad Pedagógica y Tecnológica de Colombia, realizó esta investigación que tiene como objetivo principal la recolección y adaptación de los principales textos narrativos en forma de mitos, leyendas, agüeros y refranes más representativos de la cultura popular boyacense y colombiana, basados en los requerimientos de la población beneficiaria de ELE.

Aceptando la premisa de que enseñar una lengua extranjera no solamente involucra enseñar estructuras gramaticales y léxico, sino toda una serie de componentes culturales dependiendo del entorno donde se desarrolla el proceso de enseñanza-aprendizaje, hay que considerar una gran cantidad de aspectos al preparar las actividades 0 tareas para una lección de español como lengua extranjera. Por ende, lengua y cultura, conjuntamente, comprenden el corazón de la enseñanza de una lengua extranjera. No solo se pone acento en la cultura sino también en la interculturalidad, puesto que el conocimiento de otras culturas y la convivencia en culturas diversas posibilitan enseñar y también aprender una lengua extranjera de una forma más amplia.

La propuesta de utilizar la cultura popular para enseñar español como lengua extranjera tiene como principal objetivo fortalecer la competencia comunicativa en los aprendientes de español. Por tal razón se partió de un arduo proceso investigativo focalizado en el análisis de diversos constructos teóricos provenientes de experiencias en el proceso de enseñanza y aprendizaje de ELE, que permitieron consolidar un horizonte 
teórico que llevó al diseño de talleres ajustados a las necesidades de los usuarios o hablantes de esta lengua.

Posteriormente se realizó un estudio que permitió la realimentación y elaboración de una cartilla producto de los resultados de la aplicación de una prueba piloto. Finalmente se entregó un material lo suficientemente rotundo, sustentado por artículos científicos, evidencia de todo el largo proceso de exploración en el amplio ámbito de ELE.

\section{Marco teórico}

\subsection{Cultura}

La cultura como complejidad propone la gestión entendida como la sugerencia de actos que afirman un mundo capaz de ser multiinterpretado y que siempre vuelve a iniciarse. Por eso, el hombre cuando no puede explicarse los fenómenos naturales, piensa que existen poderes externos que le señalan la explicación de lo desconocido; de ahí surgen las creencias, mitos, leyendas, agüeros y refranes, donde se manifiestan las actitudes primitivas ante la realidad del mundo circundante e interviene la magia de los fenómenos y procesos socioculturales de los pueblos.

Margulis (2011) sugiere que "la cultura se ocupa de la dimensión significativa en los fenómenos sociales: en tanto poseedores de los códigos de esa cultura, sus integrantes tienen la capacidad de leer las situaciones sociales que se les presentan" (p. 29). Por ende, el acto de lectura supone interpretar la información ligada a comportamientos, discursos y acontecimientos sociales. Pero si bien todo significa, el análisis de lo social no se agota en el plano de la significación. La cultura, entonces, sería una de las dimensiones donde podemos analizar los procesos sociales.

La dimensión cultural se ocupa del plano de la significación, de lo que es expresado con los sistemas de signos que el hombre ha creado y que median en su relación con el mundo. La cultura manifiesta su eficacia, sobre todo en el plano de la comunicación. La comunicación es el eje: la cultura es el espacio de la comunicación, un espacio social en el cual es posible comunicarse; es aptitud para comprender acciones de los demás y, hasta cierto punto, capacidad de predicción de sus comportamientos.

\subsection{Textos populares}

Los textos populares son narraciones que se transmiten, por lo general, de una generación a otra, ocasionando que cada individuo o cada grupo social aporte algo en particular; por esta razón, se les considera de autoría colectiva o anónima. Según Olvera 
(2012), dichos textos emanan del pueblo y pertenecen a la cultura porque son parte inherente de las manifestaciones cotidianas y tradiciones. Este tipo de textos se encuentra dentro de los textos recreativos que, a su vez, se dividen en dos: los textos literarios y los textos populares. Por lo anterior, los textos populares se clasifican en el refrán, el agüero, la adivinanza, el chiste, la parodia, la canción, entre otros.

\subsubsection{El refrán}

Es un enunciado breve que da una enseñanza o moraleja y se ajusta a una situación específica. De acuerdo con Uribe (2014):

los refranes o proverb and sayings en inglés, son una de las expresiones idiomáticas más universales, pues en ellos se expresa y condensa la sabiduría cotidiana, el saber popular, por lo cual su utilización, aunque con ciertas variaciones, dependiendo de las características psicográficas y geográficas de las personas, ha estado presente en todas las culturas y épocas de la humanidad. (Uribe, 2014, pp. 3-4)

El refrán exige habilidad para utilizarlo en diversas situaciones de la cotidianidad que requieren reflexión. La mayoría de las veces, los refranes representan complejidad al momento de ser comprendidos y utilizados por los aprendientes de español como lengua extranjera, ya que su significado depende en gran medida de la persona y del contexto.

\subsubsection{El agüiero}

La Real Academia Española (2014) denomina agüero al procedimiento de adivinación basado en la interpretación supersticiosa de determinadas señales, como el canto o el vuelo de las aves, los fenómenos meteorológicos, etc. Además, este presagia sucesos favorables 0 adversos, o que, a fin de cuentas, da buena o mala suerte.

Colombia es un país de supersticiones. Muchos de sus habitantes toman pequeñas y grandes decisiones basados en agüeros que fundamentan diversas creencias populares transmitidas de generación en generación y en las que creen ciegamente. En consecuencia, la comprensión de los diversos aspectos culturales que involucren el uso de agüeros dentro de un contexto real juega un aspecto importante a la hora de hacer uso de la lengua extranjera que se está aprendiendo, en este caso, el español de Colombia. 


\subsection{Textos narrativos}

Teun van Dijk (1978) define los textos narrativos como un conjunto de enunciados que contienen una estructura canónica que consta de una serie de objetos, personas o circunstancias sobre los que se quiere decir algo en un lugar y tiempo.

En Colombia existe una gran variedad de textos narrativos que reflejan la riqueza cultural de sus gentes y los pueblos o lugares que habitan. Por ende, juegan un papel importante en la elaboración de los talleres fundamentados en el uso de la cultura popular para la enseñanza del español como lengua extranjera. Los textos narrativos también involucran el acto de narrar, de contar historias, tanto verídicas como ficticias, que incluyen la descripción de acontecimientos que se desarrollan a lo largo de un espacio temporal.

Haciendo alusión a los textos narrativos que forman parte de la cultura regional y nacional colombiana, se pueden clasificar dos tipos de textos seleccionados para realizar los talleres de español como lengua extranjera:

\subsubsection{El mito}

El mito se puede definir como un relato falso u apócrifo que se asemeja al cuento, a la fábula y a la leyenda en algunos aspectos. Este tipo de texto narrativo particularmente contribuye a la educación transmisora de valores, siempre y cuando se consideren distintos puntos de vista.

Álvarez (2013) define el mito como "una tarea comprensiva que no se reduce a la filosofía, pues la interpretación de los mitos se puede realizar desde diferentes perspectivas, disciplinarias o no disciplinarias. Al final hemos de comprender que la interpretación está vinculada con una intencionalidad" (p. 81). En consecuencia, los textos narrativos de tipo mitológico constituyen una fuente inagotable de historias y relatos que pueden ser empleados con distintos fines educativos.

\subsubsection{La leyenda}

Para Timothy Tangherlini (1990), la leyenda es un relato folclórico con bases históricas, una narración tradicional corta de un solo episodio, altamente representada, realizada de modo conversacional, que refleja valores de carácter psicológico o simbólico de la creencia popular y de las experiencias colectivas, y que sirve de reafirmación de los valores comúnmente aceptados por el grupo a cuya tradición pertenece. 
Colombia cuenta con un extenso repertorio de leyendas que se transmiten de generación en generación y de un lugar a otro, y cumplen un papel importante dentro de las tradiciones orales propias de la identidad cultural del pueblo o ciudad a los que pertenecen. Generalmente, las leyendas relatan hechos asociados a misterios, a la presencia de demonios, brujas, fantasmas, objetos con poderes sobrenaturales o a espantos que no pueden ser explicados por ninguna ciencia, pero que hacen parte de la historia y se han arraigado a la cultura, porque son propios de los imaginarios colectivos de las personas.

\section{Metodología}

\subsection{Enfoque de la investigación}

La investigación se centró en la cultura popular relacionada con mitos, leyendas, agüeros y refranes. El enfoque de la investigación es de tipo cualitativo. De acuerdo con Batista (2011), se ocupa de la recolección y el análisis de información en todas sus formas posibles, donde se hacen registros narrativos de los fenómenos, trabaja con discursos de la gente, es decir, la comunicación verbal y no verbal y los estudia mediante técnicas como la observación participante. Parte de la subjetividad para encontrar el significado social, entendiéndose el investigador como parte de la realidad. Se basa en la comprensión. La relación entre sujeto y objeto es de interacción con los sujetos que estudia y busca la perspectiva interna del problema.

El método fue inductivo en la medida en que tanto para la aplicación como para el análisis de resultados se contó con la participación de un número de los estudiantes extranjeros que visitan la Universidad Pedagógica y Tecnológica de Colombia. De acuerdo con Bernal (2006), el método inductivo:

utiliza el razonamiento para obtener conclusiones que parten de hechos particulares aceptados como válidos, para llegar a conclusiones, cuya aplicación sea de carácter general. El método se inicia con un estudio individual de los hechos y se formulan conclusiones universales que se postulan como leyes, principios o fundamentos de una teoría. (Bernal, 2006, p.125)

\subsection{Participantes}

Esta investigación contó con la participación de los asistentes de Lengua Extranjera adscritos a la Escuela de Idiomas de la Universidad Pedagógica y Tecnológica de Colombia del primer semestre académico del año 2018. La muestra poblacional estuvo constituida por tres estudiantes de ELE. De acuerdo con los propósitos de esta investigación, solamente se tuvo en cuenta la participación de los sujetos que desearon colaborar volunta- 
riamente y quienes manifestaron explícitamente por escrito el interés de desarrollar los talleres.

\subsection{Selección del corpus}

Para la elaboración de los talleres que constituyen el cuerpo de la cartilla se seleccionaron los mitos, leyendas, agüeros y refranes más representativos tanto de la región en particular, como de la nación en general.

Teniendo en cuenta lo anterior, se consideró el uso de textos originales para la elaboración de los talleres. A pesar de la dificultad lingüística que se debe tener presente, una cuidadosa selección del texto por parte del profesor y el diseño de actividades pueden facilitar el trabajo, con el fin de simplificar el aprendizaje y la motivación, como afirman Molina y Ferreira (2008):

A la hora de seleccionar el texto o fragmento debemos estar seguros de que éste corresponde al nivel de la clase. Un texto demasiado complicado puede desmotivar a los estudiantes y puede crear una actitud poco receptiva ante textos literarios posteriores. Sin embargo, existen teorías según las cuales casi todos los textos podrían adaptarse a todos los niveles, la dificultad no residiría tanto en la complejidad del texto, y sí en la actividad propuesta. Nosotros decidimos. (Molina \& Ferreira, 2008, p. 675)

Para la selección de los textos se tuvieron en cuenta características como extensión del texto, claridad, uso de expresiones, coherencia y cohesión en el relato y pertinencia de la moraleja. Como resultado de la primera selección se encontraron diversas versiones de varios mitos y leyendas, ya que los contenidos cambian dependiendo de la época, origen, o editorial donde se encuentran publicados textos; se descartaron los mitos y leyendas con gran extensión de texto y se conservaron los manuscritos con mayor claridad léxica.

\subsection{Diseño de los talleres}

La información recopilada de los mitos, leyendas, refranes y agüeros contribuyó al diseño de doce unidades soportadas con teorías lingüísticas, sociolingüísticas, pedagógicas y pragmáticas, las cuales se desglosaron en: imagen de acercamiento, casilla de palabras clave, comprensión de lectura, expresión escrita, expresión oral y escucha. Esta última pretende que el estudiante identifique distintos aspectos y palabras clave que se trabajaron a lo largo de la unidad acompañados de una parte auditiva del mito o leyenda. Asimismo, cada sección de las unidades coadyuva al fortalecimiento de las competencias comunicativas que se espera que los estudiantes de español como lengua extranjera de- 
sarrollen de acuerdo con el Marco Común de Referencia para las Lenguas (MCRE). De esta manera, los procesos de enseñanza y de aprendizaje de la nueva lengua se desarrollan de manera integral.

\subsection{Estructura de las unidades}

La metodología del texto final destaca elementos y actividades de carácter comunicativo, ya que se espera incentivar la interacción en diferentes contextos donde el estudiante se exprese en lengua extranjera para manifestar sus experiencias, opiniones, gustos, sentimientos, haciendo uso de la cultura popular; de esta manera, perfecciona la estructura gramatical, enriquece su léxico y mejora su pronunciación.

En el texto se estructuraron doce unidades que tuvieron en cuenta los aspectos lingüísticos, sociales, pragmáticos e interculturales que permiten una mejor comprensión del contenido. Cada unidad consta de:

a. Imagen de acercamiento: antes de comenzar la lectura se encuentra una imagen que engloba el contenido del texto por leer. De esta manera, el estudiante extranjero es capaz de inferir de qué se trata el texto y de emitir conceptos haciendo uso de sus capacidades de interpretación.

b. Casilla de palabras clave: al costado izquierdo de cada lectura se encuentra un recuadro con diferentes palabras que mejoran la comprensión de los contenidos del texto y al mismo tiempo permiten al estudiante aumentar su léxico a la hora de utilizar las expresiones en diversas situaciones comunicativas.

c. Lectura: en las doce unidades de la cartilla se presentan los textos que contienen los mitos, leyendas, agüeros y refranes, los cuales tienen la intensión de ejercitar al estudiante en los tres niveles de lectura: literal, inferencial y sociocrítica.

d. Comprensión de lectura: los ejercicios que comprenden esta primera etapa se basan en actividades de identificación lexical, cuestionarios de selección múltiple con única respuesta, enunciados de opción falso/verdadero, entre otras.

e. Expresión escrita: para esta sección se pretende que los estudiantes interpreten el contenido de los textos desde una visión pragmática, haciendo uso de la lengua en contextos específicos. Por ende, las actividades propuestas a lo largo de esta etapa implican tanto comprensión lectora, como análisis sociocrítico 
de la lectura, partiendo de la realidad en la que el estudiante se ha desenvuelto durante su vida.

f. Expresión oral: las actividades contenidas en esta tercera etapa de ejercicios demandan un alto grado de interpretación, ya que el componente intercultural que se trabaja en esta sección le permite al estudiante, primero comprender y ser comprendido a través de la interacción y socialización de las actividades, y luego acercarse a la cultura colombiana. Asimismo, el estudiante interactúa significativamente mediante el diálogo, la narración y la argumentación.

g. Escucha: cada unidad concluye con una actividad de comprensión auditiva que comprende el documento sonoro y los ejercicios escritos basados en el texto narrativo que se trabaja en la unidad. Las actividades facilitan la aprehensión de significado y de sentido de los documentos ligados a la cultura popular por medio de la identificación y selección de imágenes, descripción de situaciones o eventos, enunciados para completar, cuestionarios de selección múltiple con única respuesta, entre otros.

\subsection{Aplicación y resultados del pilotaje}

Elaboradas las unidades, se llevó a cabo el pilotaje, que permitió abordar eficazmente los conocimientos presentados en cada uno de los talleres propuestos. Se desarrollaron las actividades previstas con cada uno de los estudiantes voluntarios. Inicialmente se controlaron las tareas a lo largo de cada sección de las unidades y se explicaron los puntos o contenidos que representaban dificultad, se hizo la respectiva corrección por parte de los autores de los talleres a los estudiantes extranjeros. Finalmente se evaluaron las lecciones y su incidencia en el proceso de investigación y en el resultado final.

Al finalizar la aplicación del pilotaje, los estudiantes extranjeros socializaron los talleres ante los autores investigadores de cada uno de los talleres, con la finalidad de evidenciar la claridad de las actividades, establecer el nivel de dificultad de los documentos y descartar o redactar actividades dependiendo del contenido de las unidades.

Dicho pilotaje también permitió concluir que el nivel de conocimiento heterogéneo de los aprendientes requería rediseñar distintas actividades comprendidas en cada una de las seis secciones que se definieron en las unidades, ya que la diferencia de procedencia, edad, nivel de lengua, e intereses de los estudiantes fue primordial para garantizar un mejor desempeño por parte de los aprendientes con respecto al uso adecuado de la lengua en cada una de las unidades. 
Para los aspectos contenidos en cada unidad se constató la diferencia en los avances obtenidos según el nivel del grupo: en términos de argumentación de forma oral y escrita, se detectaron algunas deficiencias, lo cual se evidenció en la necesidad por parte de los estudiantes de retomar y repetir los enunciados. En cuanto al nivel expresividad oral, los estudiantes estaban en condición de retomar expresiones que hacen parte de la cultura colombiana a la hora de justificar sus respuestas, demostrando así cierto nivel de comprensión relacionado con el uso contextualizado de dichas expresiones idiomáticas en determinadas situaciones comunicativas.

En lo concerniente a la lectura en voz alta, la interferencia se manifestó en la tendencia a pronunciar palabras y oraciones haciendo uso de la acentuación derivada del inglés o del chino mandarín, del mismo modo que en la tendencia a formar grupos vocálicos que no existen en español. Haciendo énfasis en el aspecto sociocultural, los estudiantes se apropiaron completamente de los contenidos presentados en cada uno de los textos extraídos de la cultura popular colombiana, contrastando la información con la cultura de su país de origen.

De esta manera, los resultados permiten recalcar la necesidad de una adecuada interacción cultual que facilite una mejor comprensión e interpretación de los aspectos característicos de cada región en Colombia. La interacción en diversos contextos permite a los estudiantes aprendientes del español como lengua extranjera expresar sus experiencias, opiniones, gustos y sentimientos, y, al mismo tiempo, utilizar estructuras gramaticales en español, enriquecer su campo lexical e interactuar en un entorno real.

Terminado el proceso del pilotaje, los investigadores hicieron los ajustes pertinentes para llevar a cabo el proceso de redacción final de cada una de las doce unidades del libro, que incluye preguntas de análisis, interpretación, proposición, argumentación y creación. También se hizo el registro sonoro final de las actividades de escucha.

Diseñado, ejecutado y autoevaluado, se presentó el manuscrito ante la oficina de la Dirección de Investigaciones (DIN) de la Universidad Pedagógica y Tecnológica de Colombia (UPTC), quien nombró pares evaluadores encargados de avalar la producción. Los pares emitieron conceptos acerca de la producción bibliográfica, los cuales fueron tenidos en cuenta para producir la versión final. Finalmente, el libro fue presentado ante el Comité Editorial, que autorizó la publicación de 200 ejemplares.

En este artículo se presenta una muestra de una unidad del documento final intitulado Enseñanza del español para extranjeros a través de la cultura popular, que 
se presentó como resultado final de la investigación del grupo de investigación Español como Lengua Extranjera (ELEX) de la Universidad Pedagógica y Tecnológica de Colombia.

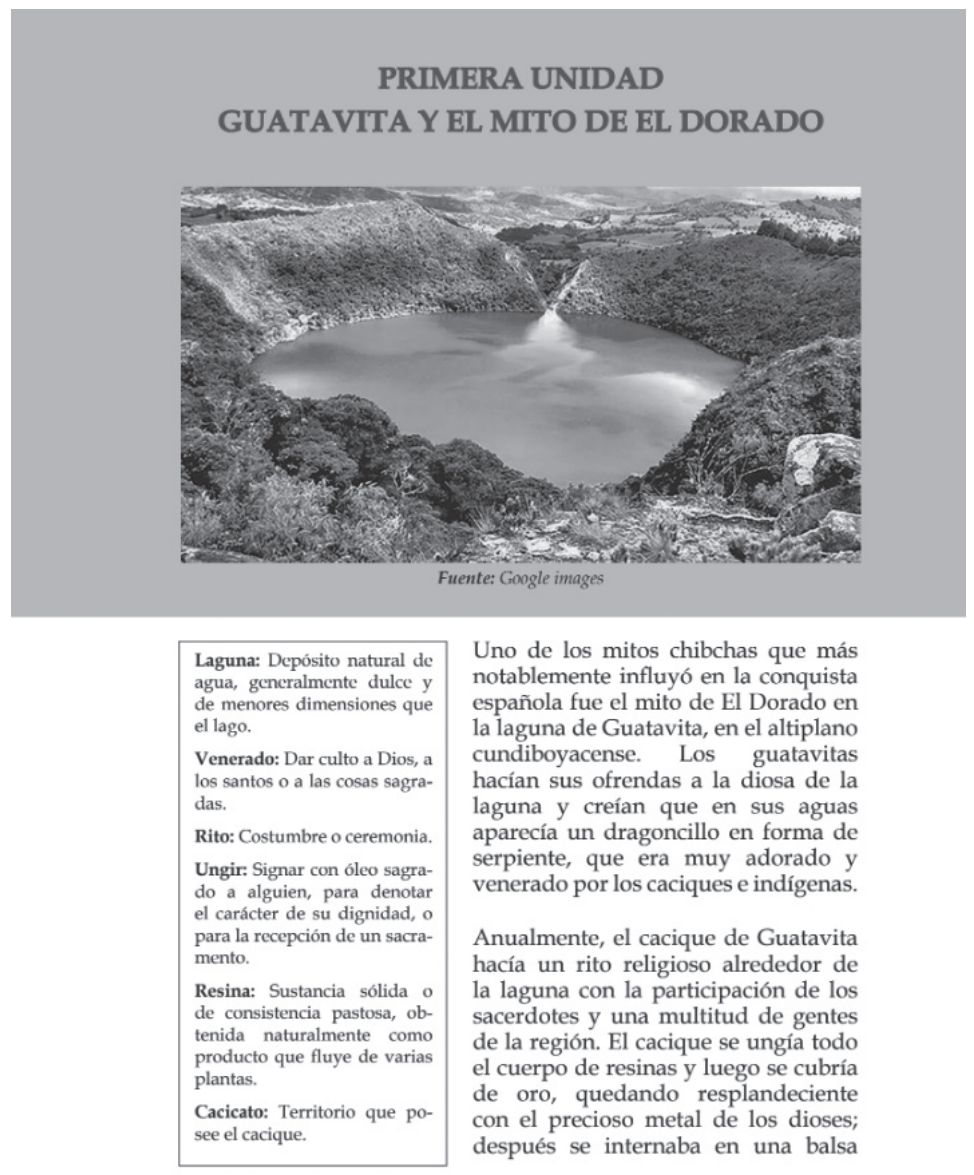

15

Figura 1. Muestra de una unidad del libro Enseñanza del español para extranjeros a través de la cultura popular.

\section{Resultados}

Como resultado del proyecto de investigación se produjo la cartilla anteriormente nombrada, la cual consta de doce unidades, cuyos títulos y estructuración de temáticas se mencionan (véase la muestra del capítulo). Estos tienen en cuenta los aspectos lingüísti- 
cos, sociales, pragmáticos e interculturales que desarrollan la competencia comunicativa del hablante extranjero.

Durante la etapa de pilotaje de los talleres, los extranjeros reforzaron sus conocimientos sobre los textos narrativos que se trabajaron. Los estudiantes afirmaron que no sabían nada sobre los diferentes mitos, leyendas, agüeros y refranes, lo que les permitió ampliar su conocimiento de la cultura colombiana. Adicionalmente, los estudiantes afirmaron que las actividades fueron llamativas y fáciles para responder, lo que permitió una mejor comprensión de cada uno de los textos. Además, se generaron diversas discusiones en cuanto a los diferentes textos narrativos que pueden ser equivalentes a los documentos y expresiones o palabras trabajadas en los talleres debido a la infinidad de similitudes que presentan.

Asimismo, los tres estudiantes voluntarios, por medio de la temática, potenciaron su competencia comunicativa haciendo uso del español, conocieron y aprendieron vocabulario desconocido, expresiones nuevas, autores y, en general, la cultura de Colombia, Boyacá y Tunja. Se espera emplear el documento final para ser trabajado con futuros estudiantes aprendientes del español como lengua extranjera que lleguen a la Universidad Pedagógica y Tecnológica de Colombia.

\section{Conclusiones}

Esta investigación permitió corroborar que los estudiantes de español como lengua extranjera son capaces de apropiarse de la cultura donde aprenden la lengua por medio de los diferentes textos narrativos que reflejan los valores, costumbres y creencias de las gentes. De igual forma, los estudiantes afirmaron que los doce talleres que se desarrollaron incentivaron su deseo de conocer y aprender más sobre las diferentes expresiones culturales que hacen parte de la colombianidad, ya que los documentos facilitaron la comprensión e interpretación de los relatos. Asimismo, los extranjeros hicieron uso de la literatura presentada como recurso para perfeccionar su nivel de comprensión y expresión en español.

Teniendo en cuenta los resultados de la investigación, la propuesta didáctica incluyó diversas actividades que permitieron a los estudiantes comprender fácilmente diferentes tipos de textos narrativos. De igual forma, con base en los talleres desarrollados por los participantes, se pudo evidenciar que es posible evitar el uso de ejercicios monótonos de gramática y léxico para que los estudiantes no sientan apatía al leer y al comprender o interpretar un documento que lo exige. 
Los aspectos lingüísticos, sociales, pragmáticos e interculturales que se tomaron en consideración a la hora de diseñar cada una de las doce unidades del libro, han facilitado la expresión de las impresiones que los estudiantes extranjeros han podido forjar acerca de la nueva cultura presentada en los talleres. De este modo, evoca en el estudiante la motivación de progresar en la comprensión de los diversos componentes lingüísticos. Cabe resaltar que los aprendientes extranjeros piden mucha información para poder justificar sus respuestas, ya sea con relación a los contenidos propuestos en los talleres 0 a tareas básicas de comunicación enfocadas en las preguntas de las unidades.

La primera parte de los talleres, que integra una imagen de acercamiento al inicio de cada unidad, permite al estudiante utilizar conocimientos previos o deducir la información que se encontrará a lo largo de la leyenda, lo que involucra el uso de la lengua extranjera haciendo uso de estrategias de descripción y narración. Por ende, dicha parte del taller representa una primera dinámica que articula la interacción entre el estudiante y el documento y propicia el proceso de adquisición y creación lingüística.

Con la ayuda de la casilla de palabras clave que se encuentra al costado izquierdo de cada lectura, los estudiantes pudieron comprender más fácilmente las palabras y expresiones colombianas que se encontraban en la leyenda, lo cual les permitió agilizar el proceso de lectura y evitar así la utilización el diccionario o recurrir al profesor para preguntar el significado de determinada palabra.

En lo concerniente a la tercera parte de los talleres de cada una de las unidades, que comprende preguntas de compresión y expresión escritural, los ejercicios estuvieron acondicionados al nivel de lengua de los estudiantes, abarcaron sobre todo ejercicios de identificación lexical, que permitieron a los aprendientes retomar el vocabulario ya presentado en la etapa de lectura. Además, se evidenció que los estudiantes no se limitaron a comprender los temas eje y el vocabulario de cada una de las leyendas, sino que al mismo tiempo hicieron asociaciones con dichos temas centrales, a través de la inferencia y el análisis de los textos desde una perspectiva sociocrítica, tanto de manera oral como escrita.

Vale la pena resaltar el impacto que las actividades de expresión oral tuvieron en el desarrollo de los talleres, pues los resultados fueron favorables debido a que el componente intercultural que se trabajó en esta sección llevó a los estudiantes a comprender y ser comprendidos a través de la interacción con los demás estudiantes y profesores, haciendo uso de la cultura colombiana. 
Para concluir, las unidades presentan un componente de comprensión auditiva. Los estudiantes sacaron partido de las grabaciones para realizar lectura guiada de las leyendas y para identificar patrones de pronunciación en español. Además, mejoraron en varios aspectos relacionados con la vocalización de diferentes sonidos fonéticos propios del castellano que no se encuentran en lenguas extranjeras como el chino-mandarín. Los estudiantes manifestaron su interés de continuar aprendiendo español con los talleres basados en la cultura popular y las contribuciones que las lecturas dejaron en su proceso de aprendizaje del español como lengua extranjera.

En definitiva, se constató que la cultura popular puede ser perfectamente aplicada como estrategia para la enseñanza y el mejoramiento del español como lengua extranjera, ya que los textos narrativos empleados para dicho fin presentan infinidad de léxico, vocabulario y expresiones utilizadas a diario y que, la mayoría de las veces, representan un gran desafío de comprensión y de expresión para los extranjeros, en diferentes situaciones de comunicación. De esta manera se potencia la competencia comunicativa de los estudiantes, quienes, a su vez, se involucran más con la cultura en la que se encuentran. Aunque gran parte de los ejercicios propuestos no representaron dificultad para los extranjeros, algunas preguntas fueron modificadas para generar respuestas mucho más reflexivas y argumentadas.

\section{Referencias}

Álvarez, A. (2013). La interpretación de los mitos desde la hermenéutica analógica. Cuicuilco, 20(58), 81. Recuperado de http://www.redalyc.org/pdf/351/35130975005.pdf

Batista, N. (2011). Proceso de la investigación cualitativa. Epistemología, metodología y aplicaciones. Bogotá: Manual Moderno.

Bernal, C. (2006). Metodología de la investigación. (2. a ed.). Bogotá: Pearson Educación.

Margulis, M. (2011). Sociología de la cultura: conceptos y problemas. Buenos Aires: Biblos.

Molina, S. \& Ferreira, J. (2008). Explotación didáctica de textos literarios en la enseñanza del español como lengua extranjera. En A. Clavero \& M. Fernández (eds.), El profesor de español LE/L2 Actas del XIX Congreso Internacional de la Asociación para la Enseñanza del Español como Lengua Extranjera (ASELE), (p. 675). Cáceres: Universidad de Extremadura.

Olvera, L. (2012, 7 de junio). Textos populares. Recuperado de http://textopopular.blogspot. com/2012/06/ 
Real Academia Española. (2014). Diccionario de la lengua española (23. ${ }^{\mathrm{a}}$ ed.). Consultado en http://www.rae.es/rae.html

Tangherlini, T. (1990). "It happened not too far from here...": a survey of legend theory and characterization. Western Folklore, 49(4), 85. https://doi.org/10.2307/1499751

Uribe, A. (2004). Los refranes: herramienta de sensibilización y formación en gerencia de servicios y mercadeo de la información. Biblios, 5(19), 3-4. Recuperado de http://www. redalyc.org/articulo.oa? $\mathrm{id}=16101910$

Van Dijk, T. (1978). La ciencia del texto. Buenos Aires: Paidós. 